\title{
A Gadolinium(III) complex based on Nitronyl nitroxide ligand exhibiting magnetic relaxation process
}

\author{
Peng $\mathrm{Hu}$, Fengping Xiao ${ }^{\mathrm{a},{ }^{*}}$ \\ Environmental and Chemical Engineering College, Zhaoqing University, Zhaoqing 526061, China. \\ afxiao7@zqu.edu.cn
}

Keywords: Lanthanides, Crystal structure, Single-ion magnet, Slow magnetic relaxation.

\begin{abstract}
Reaction of the nitronyl nitroxide radical $\mathrm{NIT}-\mathrm{CH}_{3}$ with $\mathrm{Gd}(\mathrm{hfac})_{3}$ affords a onedimensional lanthanide-nitronyl nitroxide compound: [Gd (hfac) $\left.3\left(\mathrm{NIT}-\mathrm{CH}_{3}\right)\right]_{n}$ (1) Single crystal Xray crystallographic analysis reveals that this compound is one-dimensional chain built up by Gd(hfac) 3 units bridged by radicals through their NO groups. DC magnetic susceptibilities indicate that Gd complex paramagnetic above $2.0 \mathrm{~K}$.
\end{abstract}

\section{Introduction}

Due to their potential applications in high-density information storage and quantum spintronic devices, a lot of attention has been paid to the design and construction of one-dimensional singlechain magnets (SCMs) [1-3]. SCMs are characterized as slow magnetization relaxation caused by the association of large ground state spin $\left(\mathrm{S}_{\mathrm{T}}\right)$ value with a significant uniaxial (Ising-like) magnetic anisotropy (D), which leads to a significant energy barrier to magnetization reversal (U). These kind of one-dimensional complexes can be observed magnetic hysteresis arising from slow dynamics of the magnetization of a pure 1D structure rather than 3D magnetic ordering [4].

Previous studies show that lanthanide (III) ions are good candidates for the construction of SCMs, owning to their significant magnetic anisotropy from the large and unquenched orbital angular momentum. However, the drawback for lanthanide(III) ions is the naturally accompanying quantum tunneling from the hyperfine couplings and dipolar spin-spin interactions of lanthanide ions, which always lowers the effective relaxation energy barrier and induces the loss of remnant magnetization [5]. Fortunately, exchange interactions which generally exist in molecular paramagnetic species have been proved to be an effective method to reduce quantum-tunneling relaxation and then might increase the effective relaxation energy barrier processes. One of the challenges of using this approach in lanthanide based SCMs is that the limited radial extension of the $4 \mathrm{f}$ orbital always induces weak exchange interactions. In 2011 Long research group indicated that the $\mathrm{N}_{2}{ }^{3-}$ radical ligands can effectively transfer the magnetic interactions between lanthanide ions. This strong exchange coupling between anisotropic metal and radical ligand generally leads to SCMs with high relaxation energy barrier [6].

Nitronyl nitroxides are used wildly as bridging ligands to construct one-dimensional systems because they possess two identical $\mathrm{N}-\mathrm{O}$ coordination groups, which can be coordinated to two different metal ions and contribute to compounds with one-dimensional structures. Moreover, stable radical ligands can transfer the effective magnetic interactions. Recently, SCM behavior has been observed in some nitronyl nitroxide-Co(II)/Ln(III) one-dimensional compounds, and some of them show relative high energy barrier. It's worth noting that these reported SCMs are base on aromatic group substituted nitronyl nitroxides [7-8], further research of SCMs base on aliphatic group substituted nitronyl nitroxides is neccessary. In order to further explore the relationship between the magnetic properties and substitution of the radical ligands, we report one one-dimensional complex $\left[\mathrm{Gd}(\mathrm{hfac})_{3}\left(\mathrm{NIT}-\mathrm{CH}_{3}\right)\right]_{\mathrm{n}}(\mathbf{1})\left(\mathrm{NIT}-\mathrm{CH}_{3}=2,4,4,5,5\right.$-pentamethylimidazolyl-1-oxyl-3-oxide). Magnetic studies indicate that this Gd complex paramagnetic above $2.0 \mathrm{~K}$. 


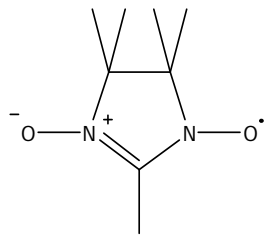

$\mathrm{NIT}-\mathrm{CH}_{3}$

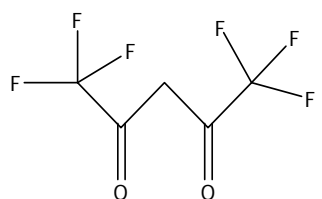

hfac

Scheme 1. Molecule structure of NIT- $\mathrm{CH}_{3}$ and hfac.

\section{Experimental Section}

\subsection{Synthesis of [Gd(hfac)3(NIT-CH3)]n.}

A solution of $\mathrm{Gd}(\mathrm{hfac})_{3} \cdot 2 \mathrm{H}_{2} \mathrm{O}(0.0814 \mathrm{~g} 0.1 \mathrm{mmol})$ in $30 \mathrm{~mL}$ dry boiling n-heptane was heated to reflux for about $1.5 \mathrm{~h}$. Then the solution was cooled to $60{ }^{\circ} \mathrm{C}$, a solution of NIT- $\mathrm{CH}_{3}(0.0171 \mathrm{~g} 0.1$ $\mathrm{mmol})$ ) in $2 \mathrm{~mL}$ of $\mathrm{CH}_{2} \mathrm{Cl}_{2}$ was added. The resulting solution was stirred for $1 \mathrm{~min}$ and cooled to room temperature. After about two days, red crystals suitable for single-crystal X-ray analysis were collected, yield $47 \%$. Anal. Calc. for $\mathrm{C}_{23} \mathrm{H}_{18} \mathrm{~F}_{18} \mathrm{~N}_{2} \mathrm{O}_{8} \mathrm{Gd}$ (949.63): calcd. C 29.09, H 1.91, N 2.95 \%; found C 29.41, H 1.98, N 3.02 \%. FT-IR (KBr): 1653 (s), 1618 (s) 1559 (s), 1532 (s), 1258 (s) 1205 (s), $1147(\mathrm{~s}), 801(\mathrm{~m}), 661(\mathrm{~m}) \mathrm{cm}^{-1}$.

\subsection{X-ray crystal structure determinations}

$\mathrm{X}$-ray single-crystal diffraction data for all four complexes were collected using a Bruker APEXII CCD diffractometer at $173 \mathrm{~K}$ equipped with graphite-monochromated $\mathrm{Mo} / \mathrm{K} \alpha$ radiation $(\lambda=$ $0.71073 \AA$ ). The structures were solved by direct methods by using the program SHELXS-97 and refined by full-matrix least-squares methods on $F^{2}$ with the use of the SHELXL-97 program package. Anisotropic thermal parameters were assigned to all non-hydrogen atoms. The hydrogen atoms were set in calculated positions and refined as riding atoms with a common fixed isotropic thermal parameter. Disordered C and F atoms were observed for all compounds. The restraints of SPLIT, DELU and ISOR were applied to keep the disordered molecules reasonable. CCDC: 1548423, 1

\section{Results and discussion}

\subsection{Structural descriptions}

As shown in Figure 1, complex 1 crystallizes in $P 2{ }_{1} / n$ space group with a monoclinic crystal system. The $\left[\mathrm{Gd}(\mathrm{hfac})_{3}\right]$ units are connected by NIT- $\mathrm{CH}_{3}$ to form a one-dimensional infinite $\mathrm{Gd}(\mathrm{III})-$ NIT- $\mathrm{CH}_{3}$ chain. There is only one crystallographically independent $\mathrm{Gd}(\mathrm{hfac})_{3}\left(\mathrm{NIT}-\mathrm{CH}_{3}\right)$ moiety in the asymmetric unit. The nitroxide ligands which connect to the same Gd(III) are trans and the angle of $\mathrm{O}_{\mathrm{rad}}-\mathrm{Gd}-\mathrm{O}_{\mathrm{rad}}$ is found to be $131.10^{\circ}$. The $\mathrm{Gd}-\mathrm{O}$ (radical) bond lengths are $2.370 \AA$ and $2.388 \AA$, respectively. The $\mathrm{Gd}-\mathrm{O}(\mathrm{hfac})$ distances are in the range of $2.358-2.378 \AA$. The nearest intrachain $\mathrm{Gd} \cdot \mathrm{Gd}$ distances are found to be $8.194 \AA$ for complex 1 .
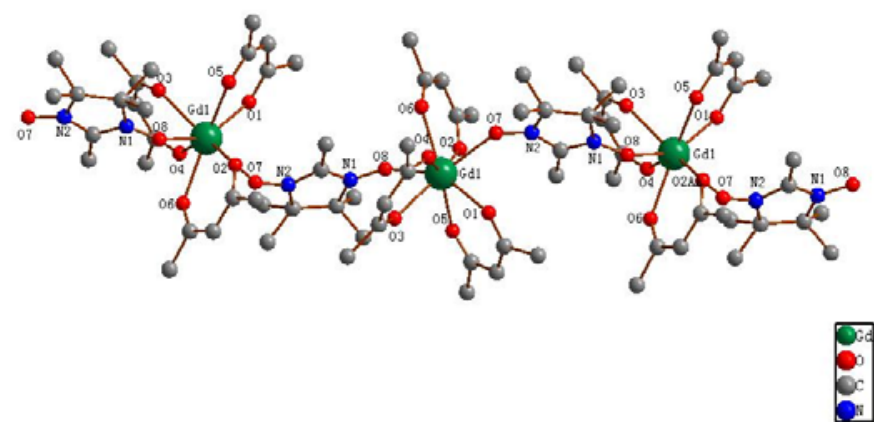

Fig. 1 One-dimensional chain structure of complex 1 with the atom-labeling scheme. All hydrogen and fluorine atoms have been omitted for clarity. 


\subsection{Magnetic properties}

To study the static magnetic properties of complex 1, variable-temperature magnetic susceptibilities were measured from $300 \mathrm{~K}$ to $2 \mathrm{~K}$ in an applied field of $1 \mathrm{kOe}$. The $\chi_{\mathrm{M}} T v s . T$ plots for 1 are shown in Figure 2 left. At $300 \mathrm{~K}$, the $\chi_{\mathrm{M}} T$ value is $8.21 \mathrm{~cm}^{3} \mathrm{~K} \mathrm{~mol}^{-1}$, close to the theoretical value of uncoupled $\mathrm{Gd}^{\mathrm{III}}$ ion $8.26 \mathrm{~cm}^{3} \mathrm{~K} \mathrm{~mol}^{-1}$ which equal to $7.88 \mathrm{~cm}^{3} \mathrm{~K} \mathrm{~mol}^{-1}$ (f $\mathrm{f}^{7}$ electron configuration) plus $0.375 \mathrm{~cm}^{3} \mathrm{~K} \mathrm{~mol}^{-1}$ (one organic radical $\mathrm{S}=1 / 2$ ). Upon cooling to $50 \mathrm{~K}$, a slow increase of $\chi_{\mathrm{M}} T$ value to $8.33 \mathrm{~cm}^{3} \mathrm{~K} \mathrm{~mol}^{-1}$ was observed, then $\chi_{\mathrm{M}} T$ increases rapidly to the value of $9.92 \mathrm{~cm}^{3} \mathrm{~K} \mathrm{~mol}^{-1}$ at $2 \mathrm{~K}$. This increase of $\chi \mathrm{M} T$ from $300 \mathrm{~K}$ to $2 \mathrm{~K}$ indicates ferromagnetic interaction in the molecule.

The field dependence of magnetization of complex 1 has been determined at $2 \mathrm{~K}$ in the range of 0-70 kOe (Figure 2 right). With increasing the applied field, $M$ increases up to $7.19 N \beta$ at $70 \mathrm{kOe}$ without reaching saturation. This may be attributed to the presence of magnetic anisotropy in the system.
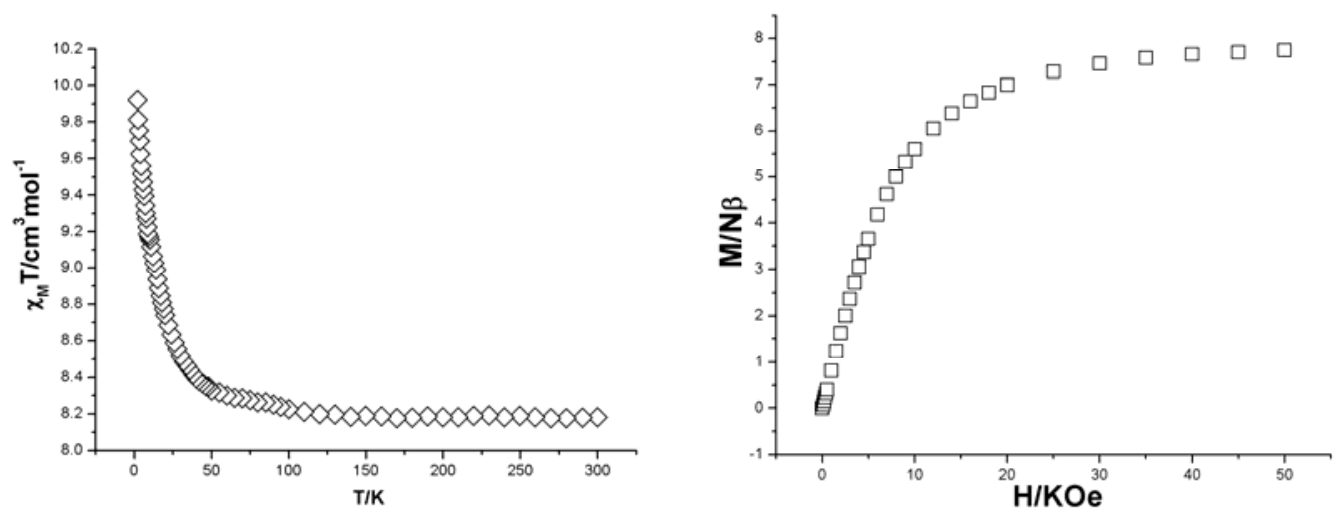

Fig. 2 Temperature dependence of $\chi_{\mathrm{M}} T$ for complex 1 (left) and field dependence of magnetization of complex 1 at $2.0 \mathrm{~K}$ (right).

\section{Summary}

In this paper, we have successfully obtained a one-dimensional Gd-radical complex base on Gd and aliphatic group substituted nitronyl nitroxides. Magnetic studies reveal that this complex paramagnetic above $2.0 \mathrm{~K}$.

\section{References}

[1]. X. Yi, G. Calvez, C. Daiguebonne, et al. Rational organization of lanthanide-based SMM dimers into three-dimensional networks. Inorg. Chem. Vol. 54 (2015) p. 5213-5129.

[2]. A. Dei, D. Gatteschi, Molecular (nano) magnets as test grounds of quantum mechanics, Angew. Chem. Int. Ed. Vol. 50 (2011) p. 11852-11858.

[3]. M. Mannini, F. Pineider, P. Sainctavit, et al. Magnetic memory of a single-molecule quantum magnet wired to a gold surface, Nat. Mater. Vol. 8 (2009) p. 194-197.

[4]. F. Troiani, M. Affronte, Molecular spins for quantum information technologies. Chem. Soc. Rev. Vol. 40 (2011) p. 3119-3129.

[5]. M. Mannini, F. Pineider, C. Danieli, et al. Quantum tunnelling of the magnetization in a monolayer of oriented single-molecule magnets, Nature Vol. 468 (2010) p. 417-421.

[6]. D. Rinehart, M. Fang, W. J. Evans, et al. Strong exchange and magnetic blocking in N-2(3-)radical-bridged lanthanide complexes, Nat. Chem. Vol. 3 (2011) p. 538-542.

[7]. F. Pointillart, Y.L. Gal, S. Golhen, et al. Paramagnetic 3D coordination complexes involving redox-active tetrathiafulvalene derivatives: an efficient approach to elaboratemulti-properties materials, Dalton Trans. Vol. 42 (2013) p. 1949-1960.

[8]. K. Bernot, L. Bogani, A. Caneschi, et al., A family of rare-earth-based single chain magnets: playing with anisotropy, J. Am. Chem. Soc. Vol. 128 (2006) p. 7947-7956. 